\title{
Complexity classification of local Hamiltonian problems
}

\author{
Toby Cubitt \\ Department of Applied Mathematics and Theoretical Physics, \\ University of Cambridge, \\ Cambridge, UK. \\ tsc25ecam.ac.uk
}

\author{
Ashley Montanaro \\ Department of Computer Science, \\ University of Bristol, \\ Bristol, UK. \\ ashleyecs.bris.ac.uk
}

\begin{abstract}
The calculation of ground-state energies of physical systems can be formalised as the $k$-LOCAL HAMILTONIAN problem, which is the natural quantum analogue of classical constraint satisfaction problems. One way of making the problem more physically meaningful is to restrict the Hamiltonian in question by picking its terms from a fixed set $\mathcal{S}$. Examples of such special cases are the Heisenberg and Ising models from condensed-matter physics.

In this work we characterise the complexity of this problem for all 2-local qubit Hamiltonians. Depending on the subset $\mathcal{S}$, the problem falls into one of the following categories: in P; NP-complete; polynomial-time equivalent to the Ising model with transverse magnetic fields; or QMA-complete. The third of these classes contains NP and is contained within StoqMA. The characterisation holds even if $\mathcal{S}$ does not contain any 1-local terms; for example, we prove for the first time QMA-completeness of the Heisenberg and $\mathrm{XY}$ interactions in this setting. If $\mathcal{S}$ is assumed to contain all 1-local terms, which is the setting considered by previous work, we have a characterisation that goes beyond 2-local interactions: for any constant $k$, all $k$-local qubit Hamiltonians whose terms are picked from a fixed set $\mathcal{S}$ correspond to problems either in $\mathrm{P}$; polynomial-time equivalent to the Ising model with transverse magnetic fields; or QMAcomplete.
\end{abstract}

These results are a quantum analogue of Schaefer's dichotomy theorem for boolean constraint satisfaction problems.

Index Terms-Hamiltonian complexity; QMA-completeness.

\section{INTRODUCTION}

Constraint satisfaction problems (CSPs) are ubiquitous in computer science and have been intensively studied since the early days of complexity theory. A beautiful and surprising result in this area is the dichotomy theorem of Schaefer [1], which completely classifies the complexity of boolean constraint satisfaction problems of a certain form. These problems can all be considered special cases of a general problem $\mathcal{S}$ CSP, where $\mathcal{S}$ is a set of constraints, each of which is a boolean function on a fixed number of bits. An instance of the problem is described by a sequence of these constraints, applied to different subsets of input bits. The task is to determine whether all the constraints can be simultaneously satisfied. For example, the 3-SAT problem fits into this class: here the constraints are disjunctions of up to 3 input bits, or their negations. Schaefer's result states that if $\mathcal{S}$ is one of a particular family of types of constraints, $\mathcal{S}$-CSP is in P; otherwise, $\mathcal{S}$-CSP is NPcomplete. This result is particularly remarkable given Ladner's theorem [2] that, assuming $\mathrm{P} \neq \mathrm{NP}$, there must be an infinite hierarchy of complexity classes between P and NP.

Schaefer's dichotomy theorem has subsequently been generalised and sharpened in a number of directions. In particular, Creignou [3] and Khanna, Sudan and Williamson [4] have completely characterised the complexity of the maximisation problem $k$-MAX-CSP for boolean constraints. Here we are again given a system of constraints, but the goal is to maximise the number of constraints we can satisfy. An example problem of this kind is MAX-CUT. A recent monograph of Creignou, Khanna and Sudan [5] has much more on this subject.

A natural quantum generalisation of constraint satisfaction problems is provided by the $k$-LOCAL HAMILTONIAN problem [6]. A $k$-local Hamiltonian is a Hermitian matrix $H$ on the space of $n$ qubits which can be written as $H=\sum_{i} H^{(i)}$, where each $H^{(i)}$ acts non-trivially on at most $k$ qubits.

Definition 1 ( $k$-LOCAL HAMILTONIAN). The (promise) problem $k$-LOCAL HAMILTONIAN is defined as follows. We are given a $k$-local Hamiltonian $H=\sum_{i=1}^{m} H^{(i)}$ on $n$ qubits with $m=\operatorname{poly}(n)$. Each $H^{(i)}$ satisfies $\left\|H^{(i)}\right\|=\operatorname{poly}(n)$ and its entries are specified by $\operatorname{poly}(n)$ bits. We are also given two rational numbers $a<b$ of $\operatorname{poly}(n)$ digits such that $b-a \geq 1 / \operatorname{poly}(n)$, and promised that the smallest eigenvalue of $H$ is either at most $a$, or at least $b$. Our task is to determine which of these two possibilities is the case.

$k$-LOCAL HAMILTONIAN is a direct generalisation of $k$ MAX-CSP; the classical problem is the special case where each matrix $H^{(i)}$ is diagonal in the computational basis and only contains 0 's and 1's. Just as $k$-MAX-CSP is NP-complete for $k \geq 2, k$-LOCAL HAMILTONIAN is QMA-complete for $k \geq 2$ [7], where QMA (quantum Merlin-Arthur) is the quantum analogue of NP [6]. If a problem is QMA-complete, this is good evidence that there is unlikely to be a polynomialtime algorithm (whether classical or quantum) to solve it.

As well as the intrinsic mathematical interest of this noncommutative generalisation of constraint satisfaction problems, a major motivation for this area is applications to physics. Indeed, the classical connection between constraint satisfaction and physics goes back at least as far as Barahona's work proving NP-hardness of cases of the Ising model [8]. One of the most important themes in condensed-matter physics 
is calculating the ground-state energies of physical systems ${ }^{1}$; for spin models, this is essentially an instance of $k$-LOCAL HAMILTONIAN.

This connection to physics motivates the study of the QMA-hardness (or otherwise) of $k$-LOCAL HAMILTONIAN with restricted types of interactions, with the aim being to prove QMA-hardness of problems of more direct physical interest, rather than the somewhat unnatural interactions that may occur in the general $k$-LOCAL HAMILTONIAN problem. This is the quantum analogue of the classical programme of proving NP-hardness of constraint satisfaction problems where the constraints are picked from a restricted set $\mathcal{S}$. One can also consider $k$-LOCAL HAMILTONIAN with restrictions on the interaction topology (for example, taking all interactions to be 2-local on a planar lattice).

In particular, it is known that 2-LOCAL HAMILTONIAN remains QMA-complete if:

- the Hamiltonian $H$ is of the Heisenberg form with arbitrary local magnetic fields,

$H=\sum_{(i, j) \in E} X_{i} X_{j}+Y_{i} Y_{j}+Z_{i} Z_{j}+\sum_{k} \alpha_{k} X_{k}+\beta_{k} Y_{k}+\gamma_{k} Z_{k}$,

where $\alpha_{k}, \beta_{k}, \gamma_{k}$ are arbitrary coefficients and $E$ is the set of edges of a 2-dimensional square lattice [9], [10];

- the Hamiltonian $H$ is of the form [11]

$$
H=\sum_{i<j} J_{i j} X_{i} X_{j}+K_{i j} Z_{i} Z_{j}+\sum_{k} \alpha_{k} X_{k}+\beta_{k} Z_{k},
$$

or

$$
H=\sum_{i<j} J_{i j} X_{i} Z_{j}+K_{i j} Z_{i} X_{j}+\sum_{k} \alpha_{k} X_{k}+\beta_{k} Z_{k},
$$

where $J_{i j}, K_{i j}, \alpha_{k}, \beta_{k}$ are arbitrary coefficients. These results determine the complexity of various special cases of the following general problem, which we call $\mathcal{S}$-HAMILTONIAN.

Definition 2 (S-HAmiltonian). Let $\mathcal{S}$ be a fixed (finite or infinite) subset of Hermitian matrices on at most $k$ qubits, for some constant $k$. The $\mathcal{S}$-HAMILTONIAN problem is the special case of $k$-LOCAL HAMILTONIAN where, for each $i$, there exists $\alpha_{i} \in \mathbb{R}$ such that $\alpha_{i} H^{(i)} \in \mathcal{S}$. That is, the overall Hamiltonian $H$ is specified by a sum of matrices $H^{(i)}$, each of which acts non-trivially on at most $k$ qubits, and whose non-trivial part is proportional to a matrix picked from $\mathcal{S}$.

We then have the following general question:

Problem 3. Given $\mathcal{S}$, characterise the computational complexity of $\mathcal{S}$-HAMILTONIAN.

We will essentially completely resolve this question in the case where every matrix in $\mathcal{S}$ acts on at most 2 qubits. Before we state our results, we observe the following important points about this problem:

\footnotetext{
${ }^{1}$ In practice, one might often actually like to determine some more complicated property of the ground state; however, calculating the energy is a reasonable starting point.
}

- In general, we assume that, given a set of interactions $\mathcal{S}$, we are allowed to produce an overall Hamiltonian by applying each interaction $M \in \mathcal{S}$ scaled by an arbitrary real weight, which can be either positive or negative. This contrasts with constraint satisfaction problems, where usually weights are restricted to be positive.

- We assume that we are allowed to apply the interactions in $\mathcal{S}$ across any choice of subsets of the qubits. That is, the interaction pattern is not constrained by any spatial locality, planarity or symmetry considerations. However, each application of a $k$-local interaction must be across a set of $k$ distinct qubits. Classically, it is common to allow one constraint in a CSP to take as input multiple copies of the same variable, but from a physical perspective this seems less meaningful so we do not allow it. Even classically, this distinctness requirement can make it more difficult to prove hardness for families of CSPs [4].

- Some of the interactions in $\mathcal{S}$ could be non-symmetric under permutation of the qubits on which they act; for example, it could make a difference whether we apply $M \in \mathcal{S}$ across qubits $(1,2)$ or qubits $(2,1)$. We assume that we are allowed to apply such interactions to any permutation of the qubits.

- We can always assume without loss of generality that the identity matrix $I \in \mathcal{S}$, as adding an arbitrarily weighted identity term (energy shift) does not change the hardness of the problem.

Making these assumptions will allow us to give a precise classification of the complexity of $\mathcal{S}$-HAMILTONIAN; the price paid is that the problem instances considered are potentially less physically meaningful (for example, containing terms with polynomially large weights, with both positive and negative signs, and with interactions across large distances). Finding a full characterisation of $\mathcal{S}$-HAMILTONIAN with additional restrictions on the form of the Hamiltonians considered seems to be a very challenging task. However, sometimes (see Section II below) we are nevertheless able to classify the complexity of $\mathcal{S}$-HAMILTONIAN even when restricted to more physically realistic interaction patterns.

A number of interesting special cases of $k$-LOCAL HAMILTONIAN which do not exactly fit into the $\mathcal{S}$-HAMILTONIAN framework have also been studied. In particular, it has been shown by Bravyi et al. [12] that $k$-LOCAL HAMILTONIAN is in the complexity class AM if the Hamiltonian is restricted to be stoquastic. A stoquastic Hamiltonian has all off-diagonal entries real and non-positive in the computational basis. Such Hamiltonians are of particular interest as they occur in a wide variety of physical systems, and also in the quantum adiabatic algorithm for SAT [13] and certain claimed implementations of quantum computation [14]. As AM is in the polynomial hierarchy, it is considered unlikely that $k$-LOCAL HAMILTONIAN with stoquastic Hamiltonians is QMA-complete. This result was subsequently sharpened by Bravyi, Bessen and Terhal [15], who showed that this problem is StoqMA-complete, where StoqMA is a complexity class which sits between 
MA and AM. On the other hand, approximating the highest eigenvalue of a stoquastic Hamiltonian is QMA-complete [16]. Bravyi and Vyalyi [17] proved that $k$-LOCAL HAMILTONIAN is in NP for 2-local Hamiltonians with commuting terms, and this has been extended recently by Hastings [18] to further classes of commuting Hamiltonians.

\section{A. Organisation and notation}

Because of space limitations, many proofs, technical details and discussions are deferred to the full version of the paper [19]. In the remainder of this extended abstract, we state our results; discuss our proof techniques; sketch the hardness proof of an key special case (the Heisenberg model); give an overview of the proof of one of our main results; and conclude with some open questions.

We use $X:=\left(\begin{array}{ll}0 & 1 \\ 1 & 0\end{array}\right), Y:=\left(\begin{array}{cc}0 & -i \\ i & 0\end{array}\right), Z:=\left(\begin{array}{cc}1 & 0 \\ 0 & -1\end{array}\right)$ to denote the Pauli matrices, and also define $\sigma^{0}:=I, \sigma^{1}:=X, \sigma^{2}:=$ $Y, \sigma^{3}:=Z$. Any $k$-qubit matrix $M$ can be decomposed as a weighted sum of tensor products of Pauli matrices. For each $\ell, 0 \leq \ell \leq k$, we call the part of $M$ corresponding to Pauli matrices which act non-trivially on exactly $\ell$ qubits the $\ell$-local part of $M$.

For any $k$-qubit matrix $M$, we let $M_{i_{1} \ldots i_{k}}$ denote the matrix formed by applying $M$ on qubits $i_{1}, \ldots, i_{k}$, tensored with the identity elsewhere. For conciseness, we usually follow the condensed-matter convention of writing $A B$ for the twoqubit matrix $A \otimes B$ (so, for example, $X X+Y Y+Z Z=$ $X \otimes X+Y \otimes Y+Z \otimes Z$ ). We usually let $n$ denote the number of qubits in the overall Hamiltonian.

\section{STATEMENT OF RESUlTS}

We begin by considering a special case of the problem, which we call $\mathcal{S}$-HAMILTONIAN WITH LOCAL TERMS, is defined as follows.

Definition 4. $\mathcal{S}$-HAMILTONIAN WITH LOCAL TERMS is the special case of $\mathcal{S}$-HAMILTONIAN where $\mathcal{S}$ is assumed to contain all 1-qubit Hermitian matrices.

That is, in the $\mathcal{S}$-HAMILTONIAN WITH LOCAL TERMS problem we are given access to all 1-local terms for free: the overall Hamiltonian is formed by taking a sum of terms from $\mathcal{S}$, each with an arbitrary positive or negative weight, then adding arbitrary 1-local terms. For any $\mathcal{S}, \mathcal{S}$-HAMILTONIAN WITH LOCAL TERMS is at least as difficult as $\mathcal{S}$-HAMILTONIAN, because it is a generalisation. It is therefore easier to prove QMA-hardness of cases of $\mathcal{S}$-HAMILTONIAN WITH LOCAL TERMS. All previous proofs of QMA-hardness of special cases of $k$-LOCAL HAMILTONIAN which we are aware of [7], [11], [9], [10] actually prove QMA-hardness of $\mathcal{S}$-HAMILTONIAN WITH LOCAL TERMS for various sets $\mathcal{S}$. Here we are able to characterise the complexity of this problem when $\mathcal{S}$ contains arbitrary matrices on up to $k$ qubits, for arbitrary $k=O(1)$.

We first need to define a notion of local diagonalisation. Let $M$ be a $k$-qubit Hermitian matrix. We say that $U \in S U(2)$ locally diagonalises $M$ if $U^{\otimes k} M\left(U^{\dagger}\right)^{\otimes k}$ is diagonal. We say that $U$ locally diagonalises $\mathcal{S}$ if $U$ locally diagonalises $M$ for all $M \in \mathcal{S}$. Note that matrices in $\mathcal{S}$ may act on different numbers of qubits, so can be of different sizes. We are now ready to state our first main result.

Theorem 5. Let $\mathcal{S}$ be an arbitrary fixed subset of Hermitian matrices on at most $k$ qubits, where $k=O(1)$. Let $\mathcal{S}^{\prime}$ be the subset formed by subtracting all 1-local terms from each element of $\mathcal{S}$, and then deleting all 0 -local matrices from the resulting set. Then:

- If $\mathcal{S}^{\prime}$ is empty, $\mathcal{S}$-HAMILTONIAN WITH LOCAL TERMS is in $\mathrm{P}$;

- Otherwise, if there exists $U \in S U(2)$ such that $U$ locally diagonalises $\mathcal{S}^{\prime}$, then $\mathcal{S}$-HAMILTONIAN WITH LOCAL TERMS is TIM-complete, where TIM is a complexity class satisfying NP $\subseteq$ TIM $\subseteq$ StoqMA (see discussion below);

- Otherwise, $\mathcal{S}$-HAMiltonian With LOCAL TERms is QMA-complete. If every matrix in $\mathcal{S}^{\prime}$ acts on 2 qubits, this holds even if we insist that the 2-qubit interactions in the final Hamiltonian are restricted to the edges of a $2 d$ square lattice and all have equal weight.

We show in the full version [19] that the condition occurring in the second case can be checked efficiently. This implies that classification of a set $\mathcal{S}$ into one of the above categories can be performed efficiently.

The alert reader may wonder why there are no NP-complete or StoqMA-complete classes in the above characterisation. In the former case, this is because of the free 1-local terms allowed. In the latter case, this is because we allow terms in $\mathcal{S}$ to be used with arbitrary weights with both signs. This implies that one can always produce a non-stoquastic Hamiltonian from any set $\mathcal{S}$ containing a non-diagonal matrix, even if all the elements of $\mathcal{S}$ have real non-positive off-diagonal entries; so stoquasticity is not a meaningful constraint in our setting. In other words, the local Hamiltonian problem restricted to the class of Hamiltonians with arbitrarily (positively or negatively) weighted stoquastic terms is QMA-complete; see the end of this section for a simple example of this.

The complexity class mentioned in the second case of Theorem 5 deserves some explanation. This picks out those special cases of $\mathcal{S}$-HAMILTONIAN WITH LOCAL TERMS which turn out to be polynomial-time equivalent to the problem of approximating the lowest eigenvalue of a Hamiltonian in the general Ising model with transverse magnetic fields. This model describes Hamiltonians of the form

$$
H=\sum_{i<j} \alpha_{i j} Z_{i} Z_{j}+\sum_{k} \beta_{k} X_{k} .
$$

Such Hamiltonians have been much studied in mathematical physics and in particular occur in the quantum adiabatic algorithm for solving optimisation problems [13]. In our terminology, the problem of determining the ground-state energy of Hamiltonians in the transverse Ising model up to inversepolynomial precision is $\{Z Z, X\}$-HAMILTONIAN; we have been unable to resist the name TIM to specify the class of problems reducible to this. Thus a promise problem $\mathcal{P}$ is in TIM if $\mathcal{P}$ can be solved by a polynomial-time Turing 
machine equipped with an oracle for the problem $\{Z Z, X\}$ HAMILTONIAN, and is TIM-hard if there is a poly-time classical reduction from $\{Z Z, X\}$-HAMILTONIAN to $\mathcal{P}$.

Fixing $\beta_{k}=0$ suffices to show that NP $\subseteq$ TIM, by the NPhardness of the general Ising model (aka MAX-CUT). In the other direction, we clearly have TIM $\subseteq$ QMA, but a tighter upper bound can be achieved. By conjugating any Hamiltonian $H$ of the form (1) by local $Z$ operations on each qubit $k$ such that $\beta_{k}>0$, which maps $X \mapsto-X$ and does not change the eigenvalues of $H, \beta_{k}$ can be assumed to be non-positive for all $k$. The resulting Hamiltonian has all off-diagonal entries nonpositive, or in other words is stoquastic [12]. Approximating the ground-state energy of stoquastic Hamiltonians is captured by the complexity class StoqMA [15], so TIM $\subseteq$ StoqMA. As StoqMA is contained within the polynomial hierarchy, and in particular within the class AM [12], it is unlikely that TIM = QMA. Thus, for sets $\mathcal{S}$ which fall into this second class, $\mathcal{S}$ HAMILTONIAN WITH LOCAL TERMS is unlikely to be QMAcomplete.

We can go further than Theorem 5, and consider a setting where we do not necessarily have access to all (or any) 1-qubit matrices. In this case, we can still completely characterise the complexity of $\mathcal{S}$-HAMILTONIAN for all sets $\mathcal{S}$ of 2qubit Hermitian matrices, with a slightly more complicated classification.

Theorem 6. Let $\mathcal{S}$ be an arbitrary fixed subset of Hermitian matrices on at most 2 qubits. Then:

- If every matrix in $\mathcal{S}$ is 1-local, $\mathcal{S}$-Hamiltonian is in $\mathrm{P}$;

- Otherwise, if there exists $U \in S U(2)$ such that $U$ locally diagonalises $\mathcal{S}$, then $\mathcal{S}$-HAMILTONIAN is NP-complete;

- Otherwise, if there exists $U \in S U(2)$ such that, for each 2-qubit matrix $H_{i} \in \mathcal{S}, U^{\otimes 2} H_{i}\left(U^{\dagger}\right)^{\otimes 2}=\alpha_{i} Z^{\otimes 2}+A_{i} I+$ $I B_{i}$, where $\alpha_{i} \in \mathbb{R}$ and $A_{i}, B_{i}$ are arbitrary singlequbit Hermitian matrices, then $\mathcal{S}$-HAMILTONIAN is TIMcomplete, where TIM is a complexity class satisfying $\mathrm{NP} \subseteq \mathrm{TIM} \subseteq$ StoqMA;

- Otherwise, $\mathcal{S}$-HAMILTONIAN is QMA-complete.

In a sense, our result completely solves Kitaev's original qubit local Hamiltonian problem [6] for the case of two-body interactions (the most physically relevant case of the original qubit local-Hamiltonian problem). We highlight some interesting special cases, which are important models in mathematical physics.

The general Heisenberg model describes Hamiltonians of the following form:

$$
\sum_{i<j} \alpha_{i j}\left(X_{i} X_{j}+Y_{i} Y_{j}+Z_{i} Z_{j}\right) .
$$

In our terminology, this corresponds to $\{X X+Y Y+Z Z\}$ Hamiltonian. By Theorem 6, finding the ground-state energy of Hamiltonians in this model is QMA-complete. Prior to this work, this problem was not even known to be NP-hard. We stress that the $\alpha_{i j}$ coefficients are allowed to be independently positive or negative; in physical systems one often restricts them to be either all positive (the antiferromagnetic case) or all negative (the ferromagnetic case); see Section IV-A for a further discussion of this point.

Schuch and Verstraete [10] previously proved QMAhardness of the Heisenberg model where arbitrary 1-local terms are also allowed, or in other words QMA-hardness of $\{X X+Y Y+Z Z\}$-HAMILTONIAN WITH LOCAL TERMS. The case where no local terms are allowed is particularly interesting because it displays a large amount of symmetry; indeed, the ground space of such a Hamiltonian on $n$ qubits must be invariant under conjugation by $U^{\otimes n}$ for arbitrary single-qubit unitaries $U$. Since the Heisenberg interaction is equivalent to projecting onto the two-qubit antisymmetric state (singlet), it can be viewed as a natural quantum generalisation of the MAX-CUT problem.

Theorem 6 also implies QMA-completeness of the XYZ and $\mathrm{XXZ}$ models in condensed-matter physics, which correspond to Hamiltonians of the form

$$
\sum_{i<j} \alpha_{i j} X_{i} X_{j}+\beta_{i j} Y_{i} Y_{j}+\gamma_{i j} Z_{i} Z_{j}
$$

The general XY model describes Hamiltonians of the following form:

$$
\sum_{i<j} \alpha_{i j}\left(X_{i} X_{j}+Y_{i} Y_{j}\right)
$$

Similarly, by Theorem 6, calculating ground state energies in this model is QMA-complete. Biamonte and Love [11] previously proved QMA-completeness if arbitrary local $X, Y$ terms are allowed and the $X_{i} X_{j}$ and $Y_{i} Y_{j}$ terms can have different weights ( $Y$ is relabelled to $Z$ in their work). It is worth remarking that, if the signs $\alpha_{i j}$ are restricted to be negative, the resulting Hamiltonian is stoquastic. Therefore, we see that QMA-completeness can be obtained from a simple special case of the stoquastic local Hamiltonian problem by allowing weights with varying signs.

Although the theory of QMA-completeness is now over a decade old [6], the list of problems proven QMA-complete is still relatively short (see [20] for a recent review). One of the original motivations for Schaefer's dichotomy theorem [1] was to make NP-hardness proofs easier, by increasing the repertoire of NP-hard problems for use in reductions. We hope that our resolution of the complexity of $\mathcal{S}$-HAMILTONIAN will be similarly useful to those wishing to prove QMA-hardness.

\section{A. Independent and subsequent work}

Shortly after a first version of this work appeared on the arXiv, Childs, Gosset and Webb [21] proved that the BoseHubbard model is QMA-complete. In proving this result, they showed that for Hamiltonians of the form

$$
\sum_{i \neq j, A_{i j}=1} X_{i} X_{j}+Y_{i} Y_{j}-\sum_{k, A_{k k}=1} Z_{k},
$$

where $A$ is the adjacency matrix of a graph, approximating the lowest eigenvalue restricted to a subspace with fixed expectation value of $Z^{\otimes n}$ ("magnetisation") is QMA-complete. 
Their work thus proves that a variant of the $\{X X+Y Y, Z\}$ HAMILTONIAN problem with an additional restriction to a subspace is QMA-complete, even if the non-zero coefficients of the terms are fixed to 1 (for $X X+Y Y$ terms) or -1 (for $Z$ terms).

More recently, Bravyi [22] has given a polynomial-time algorithm for approximating the ground-state energy of TIM Hamiltonians $H=\sum_{i \neq j} \alpha_{i j} Z_{i} Z_{j}+\sum_{k} \beta_{k} X_{k}$ in the ferromagnetic case where $\alpha_{i j} \leq 0$ for all $i \neq j$. A precise characterisation of the complexity of general Hamiltonians of this form remains open.

\section{Proof techniques}

As is typical for "dichotomy-type" results, our classification theorems proceed by identifying some special cases which are easy, and then proving hardness of all other cases. All of our hardness results are based on reductions using gadgets (as used in e.g. [7], [9], [10], [11]), rather than proving QMAhardness directly using clock constructions or similar (as used in e.g. [6], [7]).

The basic idea is to approximately simulate some set of interactions $\mathcal{A}$, where $\mathcal{A}$-HAMILTONIAN is QMA-hard, using some other set of interactions $\mathcal{B}$, thus proving QMA-hardness of $\mathcal{B}$-Hamiltonian. We use two kinds of gadgets, both analysed using perturbation theory [7]. This theory allows us to characterise the low-energy part of operators of the form $V+\Delta H$, where $V$ and $H$ are Hamiltonians and $\Delta=\operatorname{poly}(\|V\|)$ is a large coefficient. The simpler type of gadget consists of choosing a large enough constant $\Delta$ such that $V$ is effectively projected onto the ground space of $H$. This is the quantum analogue of the natural classical technique of forcing some input bits to be in a certain state by applying a heavily weighted constraint to them. A more complicated type of gadget does not have a classical analogue. Here we choose $H$ to be 1-local, and by picking somewhat smaller $\Delta$, implement an effective 2-local interaction which we did not have access to previously.

The $\mathcal{S}$-HAMILTONIAN problem contains a daunting number of cases, so the first step of our proof is to reduce the Hamiltonians we consider to a normal form by conjugating by local unitaries, which does not change the eigenvalues. It turns out that the 2-local part of any given 2-qubit Hermitian matrix $H$ which is symmetric (resp. antisymmetric) under interchange of the qubits can be reduced to a matrix of the form $\alpha X X+\beta Y Y+\gamma Z Z$ (resp. $\alpha(X Z-Z X)$ ), which drastically reduces the number of cases we need to cover. If our set $\mathcal{S}$ contains more than one interaction, we need to be careful to apply the same local unitaries to all $H \in \mathcal{S}$.

In the case of $\mathcal{S}$-HAMILTONIAN WITH LOCAL TERMS, the techniques used to prove QMA-hardness are then fairly standard (following previous work [7], [9], [10], [11]). We use our access to arbitrary 1-local terms to create perturbative gadgets which allow us to produce arbitrary interactions from interactions of the form $\alpha X X+\beta Y Y+\gamma Z Z$. In the case where $\mathcal{S}$ only contains interactions on 1 or 2 qubits, following this approach allows us to prove QMA-hardness even when all the 2-qubit interactions are equally weighted and are restricted to the edges of a $2 \mathrm{~d}$ square lattice. We can also prove QMAhardness for $k$-qubit interactions for $k>2$, which is based on using 1-local interactions to "cut out" components of the $k$-local interactions and produce 2-qubit interactions ${ }^{2}$. One interesting special case is $\mathcal{S}=\{Z Z\}$, which as discussed above is NP-hard and in StoqMA, and hence unlikely to be QMA-complete.

In the more general case of $\mathcal{S}$-HAMILTONIAN it is more difficult to prove QMA-hardness, as the lack of access to 1local terms does not allow us to use the perturbative techniques of [9], [10]. In some cases, we are also hampered by the presence of symmetry. This is highlighted by the Heisenberg model $\mathcal{S}=\{X X+Y Y+Z Z\}$. As $H=X X+Y Y+Z Z$ is invariant under conjugation by local unitaries, the same holds for the ground space of any Hamiltonian built only from $H$ terms, implying that it is hopeless to attempt to directly encode the ground state of a general Hamiltonian into a Heisenberg Hamiltonian. We therefore proceed using an encoding method where we associate a block of 3 physical qubits with a single logical qubit. This is inspired by related ideas in work on universality of the exchange interaction for quantum computation [23], but does not appear to follow from it directly. In order to make the encoding work, we use perturbation theory to effectively project onto a subspace which we can control within the 3-qubit space. An interesting aspect of the proof is that, in order to produce the correct interactions, we need to find an exactly solvable special case of the Heisenberg model with certain characteristics; very few such cases exist, but luckily the Lieb-Mattis model [24] has the properties we need.

The other important special case with significant symmetry is the $\mathrm{XY}$ model $\mathcal{S}=\{X X+Y Y\}$, which can be dealt with using similar ideas. Once these cases are proven QMAhard, it turns out that using a number of different encodings we can produce virtual interactions of either Heisenberg or $\mathrm{XY}$ type using almost any 2-qubit interaction with no 1local part, sufficing to prove QMA-hardness for these. Finally, QMA-hardness of cases with 1-local parts is proven by yet another gadget construction, this time one which removes the unwanted 1-local terms.

In many of these cases, we needed to carry out fairly complicated eigenvalue-eigenvector calculations in order to prove that our gadgets work. These calculations were performed using a computer algebra package. However, once they are found, verifying that the eigenvectors and eigenvalues are correct can easily be done by hand.

\section{EXAMPle: THE GeNeRAL HeISENBERG MODEL}

To exemplify our techniques, we begin by giving the QMA-hardness proof of one of the more interesting special cases which occur in our classification: the Heisenberg model without 1-local terms. Our proof is based on the use of the

\footnotetext{
${ }^{2}$ It is not obvious how to achieve this without having access to 1-local interactions, which is one reason why we were unable to achieve a full classification result for $\mathcal{S}$-HAMILTONIAN for $k>2$.
} 
following tool, which is similar to the "Projection Lemma" of Kempe, Kitaev and Regev [7]:

Lemma 7. Let $H$ be a Hamiltonian such that $\lambda_{\min }(H)=0$ and the next smallest non-zero eigenvalue of $H$ is 1 , and let $V$ be an arbitrary Hamiltonian such that $\|V\| \geq 1$. Further take $\Delta=\delta\|V\|^{2}$ for some $\delta \geq 4$, and let $\widetilde{H}=\Delta H+V$. Then

$$
\left\|\widetilde{H}_{<\Delta / 2}-V_{-}\right\| \leq 41 / \delta .
$$

The notation used in this lemma is as follows: $\widetilde{H}_{<\Delta / 2}$ is the restriction of $\widetilde{H}$ to the subspace spanned by eigenvectors of $\widetilde{H}$ of eigenvalue less than $\Delta / 2$, and $V_{-}=\Pi_{-} V \Pi_{-}$, where $\Pi_{-}$ is the projector onto the subspace spanned by eigenvectors of $H$ with eigenvalue 0 . Lemma 7 , which is based on underlying technical results of Oliveira and Terhal [9], improves the result of Kempe, Kitaev and Regev [7] by showing that the lowenergy subspace of $\widetilde{H}$ is actually close to $V_{-}$in operator norm, rather than the two operators just having a similar lowest eigenvalue. This will be important for us as we will need to encode data in this subspace. It is also immediate that Lemma 7 can be applied a constant number of times in series (which does not seem obvious from the result of [7]). We will use the lemma to effectively project the low-energy part of a Hamiltonian onto a smaller space, up to a small $(1 / \operatorname{poly}(n))$ additive error. For readability, we will not include these additive errors in the description that follows.

The general Heisenberg model describes Hamiltonians of the form

$$
H=\sum_{i<j} \alpha_{i j}\left(X_{i} X_{j}+Y_{i} Y_{j}+Z_{i} Z_{j}\right)
$$

Such Hamiltonians can equivalently be described in terms of the swap gate $F:=\frac{1}{2}(I+X X+Y Y+Z Z)$. Schuch and Verstraete proved that determining ground-state energies in the Heisenberg model is QMA-hard if one allows arbitrary additional 1-local terms [10]. Our task is to prove this claim without 1-local terms. The inherent symmetry of the model means that, in order to approximate an arbitrary Hamiltonian as a Heisenberg Hamiltonian, we will have to encode it somehow. In particular, we would like to encode a qubit in a larger space such that we can generate two non-commuting matrices which encode $X$ and $Z$ on the logical qubit.

The simplest such encoding possible is to associate a block of three physical qubits with each logical qubit (a similar idea was used in [23]). To take advantage of the symmetry of the swap operation, we use Schur-Weyl duality, which states that

$$
\left(\mathbb{C}^{2}\right)^{\otimes 3} \cong \mathcal{P}_{(3)} \otimes \mathcal{Q}_{(3)} \oplus \mathcal{P}_{(2,1)} \otimes \mathcal{Q}_{(2,1)}
$$

where $\mathcal{P}_{(3)}$ and $\mathcal{P}_{(2,1)}$ correspond to the irreps $(3)$ and $(2,1)$ of $S_{3}$ and $\mathcal{Q}_{(3)}$ and $\mathcal{Q}_{(2,1)}$ are irreps of $U(2)$. The point of this decomposition is that any permutation of the 3 qubits acts only on the spaces $\mathcal{P}_{(3)}, \mathcal{P}_{(2,1)} . \mathcal{P}_{(3)}$ is trivial (one-dimensional);
$\mathcal{Q}_{(3)}$ is 4-dimensional and can be written as

$$
\begin{aligned}
\mathcal{Q}_{(3)}=\operatorname{span}\{|000\rangle & , \frac{1}{\sqrt{3}}(|001\rangle+|010\rangle+|100\rangle), \\
& \left.\frac{1}{\sqrt{3}}(|110\rangle+|101\rangle+|011\rangle), \quad|111\rangle\right\} .
\end{aligned}
$$

$\mathcal{P}_{(2,1)}$ and $\mathcal{Q}_{(2,1)}$ are 2-dimensional and we have

$$
\begin{array}{r}
\mathcal{P}_{(2,1)} \otimes \mathcal{Q}_{(2,1)}=\operatorname{span}\left\{\frac{1}{\sqrt{2}}(|01\rangle-|10\rangle)|0\rangle,\right. \\
\frac{1}{\sqrt{2}}(|01\rangle-|10\rangle)|1\rangle,-\sqrt{\frac{2}{3}}|001\rangle+\frac{1}{\sqrt{6}}(|01\rangle+|10\rangle)|0\rangle, \\
\left.\sqrt{\frac{2}{3}}|110\rangle-\frac{1}{\sqrt{6}}(|01\rangle+|10\rangle)|1\rangle\right\} .
\end{array}
$$

Write $S_{1}=\mathcal{Q}_{(3)}, S_{2}=\mathcal{P}_{(2,1)} \otimes \mathcal{Q}_{(2,1)}$. Then it is clear that $F$, applied on any pair of the qubits, leaves $S_{1}$ invariant. In the case of $S_{2}$, with respect to the above basis one can explicitly calculate that

$$
F_{12}+F_{13}+F_{23}=0,-F_{12}=Z \otimes I, \frac{F_{13}-F_{23}}{\sqrt{3}}=X \otimes I .
$$

(Subscripts here denote the qubits that $F$ acts on.) On the whole space $\left(\mathbb{C}^{2}\right)^{3}$, the first of these corresponds to the projection onto $S_{1}$. Using Lemma 7 , by applying this interaction with a large but polynomially bounded weight, we can (simultaneously) enforce each of the 3-qubit blocks to be contained within $S_{2}$. Our $n$ triples of physical qubits thus give us a logical space corresponding to $n$ pairs of logical qubits; within each qubit pair we can apply $Z$ or $X$ to the first qubit. Note that these are not really separate qubits as we cannot address the second qubit.

We now need to implement interactions across pairs of logical qubits. Imagine we have two physical qubit triples, with the first triple labelled 1 to 3 , and the second triple labelled 4 to 6 . By applying $F$ operators across different pairs of physical qubits, we have 9 potential interactions on the logical space of 4 qubits, split into two blocks of two logical qubits: $(1,2)$ and $(3,4)$ (plus the 6 interactions we already know about, by applying $F$ across pairs in the same triple). By explicit calculation, each choice $(i, j)$ such that $i$ and $j$ are in different triples turns out to give a logical interaction of the form $M_{13}^{(i, j)}(2 F-I)_{24}+I^{\otimes 4} / 2$. As usual, we can ignore the identity term. We will not write out all of the matrices $M^{(i, j)}$, merely recording that

$$
\begin{gathered}
\frac{3}{2}\left(M^{(1,4)}-M^{(1,5)}-M^{(2,4)}+M^{(2,5)}\right)=X X \\
\frac{1}{2}\left(M^{(1,4)}+M^{(1,5)}-2 M^{(1,6)}+M^{(2,4)}+M^{(2,5)}\right. \\
\left.\quad-2 M^{(2,6)}-2 M^{(3,4)}-2 M^{(3,5)}+4 M^{(3,6)}\right)=Z Z
\end{gathered}
$$

and

$$
2 \sum_{i=1}^{3} \sum_{j=4}^{6} M^{(i, j)}=I I
$$


The first two of these mean that we can implement the interactions $X X$ and $Z Z$ across logical qubits $(1,3)$ - but product with $(2 F-I \otimes I)$ across qubits $(2,4)$. In other words, we can implement a logical Hamiltonian of the form

$\sum_{i=1}^{n}\left(\alpha_{i} X_{i}+\beta_{i} Z_{i}\right) I_{i^{\prime}}+\sum_{i<j}\left(\gamma_{i j} X_{i} X_{j}+\delta_{i j} Z_{i} Z_{j}\right)(2 F-I)_{i^{\prime} j^{\prime}}$,

where we identify the $i$ 'th logical qubit pair with indices $\left(i, i^{\prime}\right)$. We would like to eliminate the unwanted $(2 F-I)$ operators. One way to do this is to force the primed qubits to be in a particular state by very strong $F_{i^{\prime} j^{\prime}}$ interactions. Consider adding in the (logical) term

$$
G=\Delta \sum_{i<j} w_{i j} F_{i^{\prime} j^{\prime}}
$$

where $w_{i j}$ are some weights and $\Delta$ is very large. We can do this because we can make $I_{1} I_{3}(2 F-I)_{24}$, as shown in (2). If the ground state $|\psi\rangle$ of $G$ is non-degenerate, by Lemma 7 the primed qubits will all be effectively projected onto the ground state, and $H$ will become

$\sum_{i=1}^{n}\left(\alpha_{i} X_{i}+\beta_{i} Z_{i}\right)+\sum_{i<j}\left(\gamma_{i j} X_{i} X_{j}+\delta_{i j} Z_{i} Z_{j}\right)\left\langle\psi\left|(2 F-I)_{i^{\prime} j^{\prime}}\right| \psi\right\rangle$.

We therefore need to find a $G$ whose ground state is nondegenerate and $\left\langle\psi\left|(2 F-I)_{i^{\prime} j^{\prime}}\right| \psi\right\rangle \neq 0$ for all $i, j$ (and also these quantities should be easily computable). In particular, this implies that for all subsets $S$ of two qubits, we need $\psi_{S} \neq I / 4$. In order to find such a $G$, we study exactly solvable restricted special cases of the Heisenberg model. A model (i.e. family of Hamiltonians) is said to be exactly solvable if the eigenvalues and corresponding eigenvectors of any Hamiltonian in the model can be calculated efficiently. Only very few restricted versions of the Heisenberg model are known to be exactly solvable. The case which we will use is the Lieb-Mattis model on $n$ qubits [24]:

$$
H=\sum_{i \in A, j \in B} X_{i} X_{j}+Y_{i} Y_{j}+Z_{i} Z_{j}
$$

where $A$ and $B$ are disjoint subsets of qubits. That is, the interaction graph of this model is the complete bipartite graph on $A \times B$. For the case $|A|=|B|=n$, we have the following lemma, which combines results stated (for example) in [24], [25]. First define

$$
\left|\psi_{k}^{n}\right\rangle:=\frac{1}{\sqrt{\left(\begin{array}{l}
n \\
k
\end{array}\right)}} \sum_{x \in\{0,1\}^{n},|x|=k}|x\rangle .
$$

Lemma 8. Write

$$
H_{L M}:=\sum_{i=1}^{n} \sum_{j=n+1}^{2 n} M_{i j}=\sum_{i=1}^{n} \sum_{j=n+1}^{2 n} X_{i} X_{j}+Y_{i} Y_{j}+Z_{i} Z_{j} .
$$

Then the ground state of $H_{L M}$ is unique and given by

$$
\left|\phi_{L M}\right\rangle:=\frac{1}{\sqrt{n+1}} \sum_{k=0}^{n}(-1)^{k}\left|\psi_{k}^{n}\right\rangle\left|\psi_{n-k}^{n}\right\rangle .
$$

For $i$ and $j$ such that $1 \leq i, j \leq n$ or $n+1 \leq i, j \leq 2 n$, $\left\langle\phi_{L M}\left|F_{i j}\right| \phi_{L M}\right\rangle=1$. Otherwise, $\left\langle\phi_{L M}\left|F_{i j}\right| \phi_{L M}\right\rangle=-2 / n$.

The beautiful proof of Lemma 8 is well-known in the condensed-matter theory literature, and the most difficult part (proving uniqueness) was already shown by Lieb and Mattis in their original paper [24]. However, the ingredients of the proof are somewhat scattered, so we present a self-contained proof in the full version [19]. Given Lemma 8 and the above discussion, QMA-hardness of the Heisenberg model is essentially immediate. We first (potentially) add one triple of physical qubits to make the total number of logical qubits equal to $2 n$ for integer $n$. Then, by Lemma 7, we can effectively implement Hamiltonians of the form

$$
\begin{aligned}
& \sum_{k=1}^{2 n} \alpha_{k} X_{k}+\beta_{k} Z_{k} \\
& \quad+\sum_{i<j}\left(\gamma_{i j} X_{i} X_{j}+\delta_{i j} Z_{i} Z_{j}\right)\left\langle\phi_{L M}\left|(2 F-I)_{i^{\prime} j^{\prime}}\right| \phi_{L M}\right\rangle .
\end{aligned}
$$

As $\left\langle\phi_{L M}\left|(2 F-I)_{i^{\prime} j^{\prime}}\right| \phi_{L M}\right\rangle$ is non-zero, at most inversepolynomially small, and efficiently computable for all pairs $i, j$, by rescaling $\gamma_{i j}$ and $\delta_{i j}$ appropriately, we can effectively implement any Hamiltonian of the form

$$
\sum_{k=1}^{2 n} \alpha_{k} X_{k}+\beta_{k} Z_{k}+\sum_{i<j} \gamma_{i j} X_{i} X_{j}+\delta_{i j} Z_{i} Z_{j}
$$

for any choices of $\alpha_{k}, \beta_{k}, \gamma_{i j}, \delta_{i j}$. This suffices for QMAcompleteness [11]. We have proven the following lemma.

Lemma 9. $\{X X+Y Y+Z Z\}$-HAMILTONIAN is QMAcomplete.

\section{A. QMA-hardness with physically realistic interactions?}

Our construction proving QMA-hardness of the general Heisenberg model involves interactions between many pairs of spatially distant qubits, and also a highly non-planar interaction graph. It is natural to wonder whether one could modify it to be more physically natural, and perhaps only involving interactions on a $2 \mathrm{~d}$ square lattice, as can be achieved for $\{X X+Y Y+Z Z\}$-HAMILTONIAN WITH LOCAL TERMS [10]. The following observation (which was already made in [12] and essentially even in [24]) shows that such a QMA-hardness construction is unlikely to work for either ferromagnetic or antiferromagnetic cases.

Observation 10. Consider a Hamiltonian $H$ of the form $H=\sum_{i<j} \alpha_{i j}\left(X_{i} X_{j}+Y_{i} Y_{j}+Z_{i} Z_{j}\right)$. Then, if $\alpha_{i j} \leq 0$ for all $i$, $j$, determining the ground-state energy of $H$ up to inverse-polynomial precision is in P. If $\alpha_{i j} \geq 0$ for all $i, j$, and the graph of interactions that occur in $H$ is bipartite, determining the ground-state energy of $H$ up to inversepolynomial precision is in StoqMA.

Proof. In the first case, the ground state of $H$ is the product state $|0\rangle^{\otimes n}$, so the problem is trivial. In the second case, split the qubits on which $H$ acts into two sets $A$ and $B$ such that all interactions are between $A$ and $B$, and apply $Z$ rotations to the 
$B$ set. This corresponds to mapping every term in $H$ to a term of the form $\alpha_{i j}\left(-X_{i} X_{j}-Y_{i} Y_{j}+Z_{i} Z_{j}\right)$. This is a stoquastic matrix (i.e. all its off-diagonal entries are non-positive), so finding its ground-state energy is in StoqMA [15].

\section{The General $\mathcal{S}$-HAMiltonian PROBLEM}

Our characterisation of the complexity of the general $\mathcal{S}$ HAMILTONIAN problem will be greatly facilitated by the ability to transform any two-qubit Hermitian matrix $H$ into a standard normal form using conjugation by single-qubit unitaries. The normal form we use is essentially the same as one well-known in entanglement theory (e.g. [26]), except that we insist that the unitaries applied are the same on each qubit. This is important because mapping $H \mapsto U^{\otimes 2} H\left(U^{\dagger}\right)^{\otimes 2}$ does not change the eigenvalues of any Hamiltonian produced only from applications of $H$, as

$$
\sum_{i \neq j} \alpha_{i j}\left(U^{\otimes 2} H\left(U^{\dagger}\right)^{\otimes 2}\right)_{i j}=U^{\otimes n}\left(\sum_{i \neq j} \alpha_{i j} H_{i j}\right)\left(U^{\dagger}\right)^{\otimes n} .
$$

Any traceless two-qubit Hermitian matrix $H$ can be written as

$$
H=\sum_{i, j=1}^{3} M_{i j} \sigma^{i} \otimes \sigma^{j}+\sum_{k=1}^{3} v_{k} \sigma^{k} \otimes I+w_{k} I \otimes \sigma^{k}
$$

for some coefficients $M_{i j}, v_{k}, w_{k}$. Write $M(H)$ for the $3 \times 3$ matrix $M$ occurring in this decomposition. Also define the Pauli rank of $H$ to be the rank of $M(H)$. We observe that, if $H$ is symmetric (resp. antisymmetric) under exchange of the two qubits on which it acts, $M(H)$ is a symmetric (resp. skew-symmetric) matrix. For any $\mathcal{S}$-HAMILTONIAN problem, we can assume that every 2-qubit matrix $H \in \mathcal{S}$ is either symmetric or antisymmetric under interchange of the two qubits on which it acts. This holds because, given access to $H$, we can implement the two matrices $H^{+}=(H+F H F) / 2$ and $H^{-}=(H-F H F) / 2$, where $F$ is the swap operator, simply by applying $H$ in both the normal direction and in reverse. $\mathrm{H}^{+}$is symmetric, and $H^{-}$is antisymmetric. We have lost nothing by doing this, as $H^{+}+H^{-}=H$.

Lemma 11. Let $H$ be a traceless 2-qubit Hermitian matrix. If $H$ is symmetric under exchanging the two qubits on which it acts, there exists $U \in S U(2)$ such that

$$
U^{\otimes 2} H\left(U^{\dagger}\right)^{\otimes 2}=\sum_{i=1}^{3} \alpha_{i} \sigma^{i} \otimes \sigma^{i}+\sum_{j=1}^{3} \beta_{j}\left(\sigma^{j} \otimes I+I \otimes \sigma^{j}\right),
$$

for some real coefficients $\alpha_{i}, \beta_{j}$. If $H$ is antisymmetric under this exchange, there exists $U \in S U(2)$ and $i \neq j$ such that

$U^{\otimes 2} H\left(U^{\dagger}\right)^{\otimes 2}=\alpha\left(\sigma^{i} \otimes \sigma^{j}-\sigma^{j} \otimes \sigma^{i}\right)+\sum_{k=1}^{3} \beta_{k}\left(\sigma^{k} \otimes I-I \otimes \sigma^{k}\right)$,

for some real coefficients $\alpha, \beta_{k}$.

Lemma 11 allows us to reduce the complexity of the $\mathcal{S}$ HAMILTONIAN problem to a manageable number of special cases. As well as the Heisenberg model discussed above, we prove QMA-completeness of the XY model and a skewsymmetric case.

Lemma 12. $\{X X+Y Y\}$-HAMILTONIAN is QMA-complete.

Lemma 13. $\{X Z-Z X\}$-HAMILTONIAN is QMA-complete.

Based on reductions from these special cases, we can prove QMA-completeness more generally:

Lemma 14. For any real $\beta, \gamma$ such that at least one of $\beta$ and $\gamma$ is non-zero, $\{X X+\beta Y Y+\gamma Z Z\}$-HAMILTONIAN is QMA-complete.

Lemma 15. For any $\beta, \gamma$ such that at least one of $\beta$ and $\gamma$ is non-zero, and any single-qubit Hermitian matrix $A,\{X X+$ $\beta Y Y+\gamma Z Z+A I+I A\}$-HAMILTONIAN is QMA-complete.

Lemma 16. For any single-qubit Hermitian matrix $A,\{X Z-$ $Z X+A I-I A\}$-HAMILTONIAN is QMA-complete.

We will also need to consider some cases which are unlikely to be QMA-complete:

Lemma 17. $\{Z Z\}$-HAMILTONIAN WITH LOCAL TERMS is TIM-complete.

Lemma 18. For any single-qubit Hermitian matrix $A$ such that $A$ does not commute with $Z,\{Z Z, X, Z\}$-HAMILTONIAN reduces to $\{Z Z+A I+I A\}$-HAMILTONIAN.

Lemma 19. For any single-qubit Hermitian matrix $A$ such that $A$ does not commute with $Z,\{Z Z, X, Z\}$-HAMILTONIAN reduces to $\{Z Z, A I-I A\}$-HAMILTONIAN.

Finally, we consider the purely classical case of diagonal matrices.

Lemma 20. Let $\mathcal{S}$ be a set of diagonal Hermitian matrices on at most 2 qubits. Then, if every matrix in $\mathcal{S}$ is 1-local, $\mathcal{S}$ Hamiltonian is in P. Otherwise, $\mathcal{S}$-Hamiltonian is NP. complete.

Based on all the above lemmas, we are ready to prove Theorem 6 , which we restate as follows.

Theorem 6 (restated). Let $\mathcal{S}$ be an arbitrary fixed subset of Hermitian matrices on at most 2 qubits. Then:

- If every matrix in $\mathcal{S}$ is 1-local, $\mathcal{S}$-HAMILTONIAN is in $\mathrm{P}$;

- Otherwise, if there exists $U \in S U(2)$ such that $U$ locally diagonalises $\mathcal{S}$, then $\mathcal{S}$-HAMILTONIAN is NP-complete;

- Otherwise, if there exists $U \in S U(2)$ such that, for each 2-qubit matrix $H_{i} \in \mathcal{S}, U^{\otimes 2} H_{i}\left(U^{\dagger}\right)^{\otimes 2}=\alpha_{i} Z^{\otimes 2}+A_{i} I+$ $I B_{i}$, where $\alpha_{i} \in \mathbb{R}$ and $A_{i}, B_{i}$ are arbitrary singlequbit Hermitian matrices, then $\mathcal{S}$-HAMILTONIAN is TIMcomplete, where NP $\subseteq$ TIM $\subseteq$ StoqMA;

- Otherwise, $\mathcal{S}$-HAMILTONIAN is QMA-complete.

Proof. The first case is clear: any Hamiltonian that can be made from $\mathcal{S}$ is of the form $H=\sum_{i} H_{i}$ for 1-local matrices $H_{i}$, so the lowest eigenvalue of $H$ is the sum of the lowest eigenvalues of the individual matrices $H_{i}$, which can be 
calculated efficiently. For the second case, if there exists such a $U$, applying it gives a set of diagonal matrices where at least one is not 1-local (or we would be in the first case). The claim then follows from Lemma 20.

For the third case, the problem is clearly no harder than $\{Z Z\}$-HAMILTONIAN WITH LOCAL TERMS, so is contained within TIM by Lemma 17. To prove TIM-hardness, first note that after applying $U$, there must exist a matrix $H_{i} \in \mathcal{S}$ of the form $\alpha_{i} Z Z+A_{i} I+I B_{i}$ with $\alpha_{i} \neq 0$, or we would be in the first case. Symmetrising and rescaling, we can make a matrix of the form $Z Z+\beta(A I+I A)$ (where $\beta$ or $A$ might be zero). If $A$ does not commute with $Z$, Lemma 18 implies that $\{Z Z, X, Z\}$-Hamiltonian reduces to $\mathcal{S}$-Hamiltonian, so $\mathcal{S}$-HAMILTONIAN is TIM-hard. So assume that $A$ commutes with $Z$. As $A$ can be taken to be traceless by adding an overall identity term, this is equivalent to $A$ being proportional to $Z$. As we are not in the second case, there must also either exist a 2-qubit matrix $H_{j} \in \mathcal{S}$ of the form $\alpha_{j} Z Z+A_{j} I+I B_{j}$, where either $A_{j}$ or $B_{j}$ does not commute with $Z$, or a 1-qubit matrix $H_{k} \in \mathcal{S}$ that does not commute with $Z$. If the latter possibility is true, we can make $I H_{k}+H_{k} I$, so it suffices to assume the former is true. Note that possibly $i=j$ or $\alpha_{j}=0$ (but not both)

First assume that $A_{j} \neq-B_{j}$. Then by rescaling and symmetrising, we can assume we have access to matrices of the form $H_{i}=Z Z+\alpha(Z I+I Z), H_{j}=\gamma Z Z+B I+I B$, where $B$ is a traceless Hermitian matrix that does not commute with $Z$, and $\alpha, \gamma \in \mathbb{R}$. By adding a suitable multiple of $H_{i}$ to $H_{j}$ and rescaling, we can produce a matrix $H^{\prime}$ such that $H^{\prime}=Z Z+A I+I A$ for some matrix $A$ which does not commute with $Z$. By Lemma 18 , this implies that $\{Z Z, X, Z\}$-Hamiltonian reduces to $\mathcal{S}$-Hamiltonian, so once again $\mathcal{S}$-HAMILTONIAN is TIM-hard.

On the other hand, if $A_{j}=-B_{j}$, we have $H_{i}=Z Z+$ $\alpha(Z I+I Z), H_{j}=\gamma Z Z+B I-I B$, where $B$ is a traceless Hermitian matrix that does not commute with $Z$, and $\alpha, \gamma \in \mathbb{R}$. By adding a suitable multiple of $H_{i}$ to $H_{j}$, antisymmetrising and rescaling, we can produce a matrix $Z Z+B I-I B$ for some $B$ that does not commute with $Z$. Lemma 19 then implies that $\mathcal{S}$-HAMILTONIAN is TIM-hard.

We finally address the fourth case (the QMA-hard case), which is split into two subcases. In the first subcase, assume there exists at least one 2-qubit matrix $H \in \mathcal{S}$ which has Pauli rank at least 2. $M(H)$ can be assumed to be either symmetric or skew-symmetric. If $M(H)$ is symmetric, by Lemma 11 (and possibly relabelling Pauli matrices), using local unitaries $H$ can be mapped to $X X+\beta Y Y+\gamma Z Z+A I+I A$ for some $\beta, \gamma$ such that at least one of them is non-zero and some single-qubit Hermitian matrix $A$, so QMA-completeness follows from Lemma 15. If $M(H)$ is skew-symmetric, we get QMA-completeness from Lemma 16.

In the second subcase, assume all 2-qubit matrices in $\mathcal{S}$ have Pauli rank 1. There does not exist $U$ such that $U^{\otimes 2} H_{i}\left(U^{\dagger}\right)^{\otimes 2}=\alpha_{i} Z^{\otimes 2}+A_{i} I+I B_{i}$ for all $H_{i} \in \mathcal{S}$, otherwise we would be in the third case. So in this subcase there must exist a pair $i \neq j$ and a unitary $U$ such that
$U^{\otimes 2} H_{i}^{(2)}\left(U^{\dagger}\right)^{\otimes 2}$ is diagonal, but $U^{\otimes 2} H_{j}^{(2)}\left(U^{\dagger}\right)^{\otimes 2}$ is not, where $H_{i}^{(2)}$ is the 2-local part of $H_{i}$. By applying this $U$ and rescaling, we can assume that $H_{i}=Z Z+\delta(A I+I A)$, $H_{j}=(\alpha X+\beta Y+\gamma Z)^{\otimes 2}+\eta(B I+I B)$, for some real $\alpha, \beta, \gamma, \delta, \eta$ where at least one of $\alpha$ or $\beta$ is non-zero. So, by rescaling $H_{j}$, we can assume that $\alpha^{2}+\beta^{2}=1$. There exists an $\mathrm{SO}(3)$ rotation $R$ which maps $(\alpha, \beta, \gamma)$ to $(1,0, \gamma)$ while leaving $(0,0,1)$ unchanged. Therefore, there exists a unitary $V$ such that $V^{\otimes 2} H_{j}\left(V^{\dagger}\right)^{\otimes 2}=(X+\gamma Z)^{\otimes 2}$ and also $V^{\otimes 2} H_{i}^{(2)}\left(V^{\dagger}\right)^{\otimes 2}=Z Z$. If $\gamma=0$, we have something of the form $H_{i}=Z Z+\delta(A I+I A), H_{j}=X X+\eta(B I+I B)$. Adding these two matrices, relabelling Pauli matrices and using Lemma 12 and Lemma 15, this case is also QMAcomplete. If $\gamma \neq 0$, by rescaling and subtracting $H_{i}$ from $H_{j}$, we can make a matrix whose 2-local part is unitarily equivalent to $X X+\gamma^{\prime} Z Z$ for some $\gamma^{\prime} \neq 0$, so this case is QMA-complete by Lemmas 14 and 15 .

\section{OUTLOOK}

We have completely resolved the complexity of a natural subclass of $\mathcal{S}$-HAMILTONIAN problems. However, many interesting generalisations and open problems remain, e.g.:

1) Can we generalise our results to $k$-local Hamiltonians for $k>2$ ? Although we achieved this for $\mathcal{S}$-HAMILTONIAN WITH LOCAL TERMS, one potentially significant difficulty with improving this to the full $\mathcal{S}$-HAMILTONIAN problem is that no comparable normal form exists for Hermitian matrices on $k \geq 3$ qubits. Another issue is that reduction to the 2-local case, which we used for $\mathcal{S}$-HAMILTONIAN WITH LOCAL TERMS, does not seem easy to perform without having access to 1-local terms.

2) Can we generalise our results beyond qubits? Again, this could be difficult as the equivalent generalisation of Schaefer's dichotomy theorem [1] to constraint satisfaction problems on a 3 -element domain took 24 years, being resolved in 2002 by Bulatov [27].

3) Can we prove hardness, or otherwise, for more restricted types of Hamiltonian? One way of restricting further would be to put limitations on the signs or types of coefficients allowed (such as the antiferromagnetic Heisenberg model), another would be to restrict the topology of interactions (such as only allowing a planar graph, or a square lattice). We were able to achieve the latter for 2-qubit interactions with arbitrary 1-local terms, but this seems more difficult for the general $\mathcal{S}$-HAMiLtonian problem (but see Observation 10 for a small step in this direction).

Another case which has been of interest is Hamiltonians whose terms commute pairwise. In this case the $k$-local Hamiltonian problem is in $\mathrm{P}$ for various special cases: 2local Hamiltonians [17], 3-local qubit Hamiltonians [28], and $k$-local Hamiltonians whose terms are projectors onto eigenspaces of Pauli matrices [29]. Another example in this vein is a result of Schuch proving that the problem is in NP for a special class of commuting 4-local qubit Hamiltonians [30]. 
4) We were not able to completely determine the complexity of the class of $\mathcal{S}$-HAMILTONIAN problems which are polynomial-time equivalent to the Ising model with transverse magnetic field, merely encapsulating them by a new complexity class TIM, where NP $\subseteq$ TIM $\subseteq$ StoqMA. Could one of these inclusions be an equality? Our intuition is that at least the inclusion MA $\subseteq$ TIM should hold, but thus far the proof has eluded us.

5) Our results can be seen as a quantum generalisation of dichotomy theorems for the $k$-MAX-CSP problem [5]. Another way to generalise Schaefer's original dichotomy theorem [1] would be to prove a similar result for the quantum $k$-SAT problem. This is a variant of $k$-LOCAL HAMILTONIAN where each term is a projector, and we ask whether there exists a state which is in the nullspace of all the projectors ("satisfies all the constraints").

6) In a different direction, an interesting open question is whether one can prove a dichotomy theorem for unitary quantum gates. For example, given a set $\mathcal{G}$ of unitary gates, are circuits made up of gates picked from $\mathcal{G}$ always either classically simulable or universal for BQP? This question was resolved quite recently for gates produced by applying 2-local Hamiltonians from a given set for arbitrary lengths of time [31]. The general question is likely to be sensitive to the precise definitions of "simulable" and "universal", as demonstrated by the apparently intermediate class of commuting quantum computations [32].

\section{ACKNOWLEDGMENTS}

Some of this work was completed while AM was at the University of Cambridge. TC is supported by the Royal Society. AM would like to thank Mick Bremner for pointing out reference [23], and we would like to thank various referees for their helpful comments.

\section{REFERENCES}

[1] T. Schaefer, "The complexity of satisfiability problems," in Proc. $10^{\text {th }}$ Annual ACM Symp. Theory of Computing, 1978, pp. 216-226.

[2] R. Ladner, "On the structure of polynomial time reducibility," J. ACM, vol. 22, no. 1, pp. 155-171, 1975.

[3] N. Creignou, "A dichotomy theorem for maximum generalized satisfiability problems," J. Comput. Syst. Sci., vol. 51, no. 3, pp. 511-522, 1995.

[4] S. Khanna, M. Sudan, and D. Williamson, "A complete classification of the approximability of maximization problems derived from Boolean constraint satisfaction," in Proc. $29^{\text {th }}$ Annual ACM Symp. Theory of Computing, 1997, pp. 11-20.

[5] N. Creignou, S. Khanna, and M. Sudan, Complexity Classifications of Boolean Constraint Satisfaction Problems. SIAM, 2001.

[6] A. Y. Kitaev, A. H. Shen, and M. N. Vyalyi, Classical and Quantum Computation, ser. Graduate Studies in Mathematics. AMS, 2002, vol. 47.

[7] J. Kempe, A. Kitaev, and O. Regev, "The complexity of the local Hamiltonian problem," SIAM J. Comput., vol. 35, no. 5, pp. 1070-1097, 2006, quant-ph/0406180.

[8] F. Barahona, "On the computational complexity of Ising spin glass models," J. Phys. A: Math. Gen., vol. 15, no. 10, p. 3241, 1982.

[9] R. Oliveira and B. Terhal, "The complexity of quantum spin systems on a two-dimensional square lattice," Quantum Inf. Comput., vol. 8, no. 10, pp. 0900-0924, 2008, quant-ph/0 004050 .
[10] N. Schuch and F. Verstraete, "Computational complexity of interacting electrons and fundamental limitations of Density Functional Theory," Nature Physics, vol. 5, pp. 732-735, 2009, arXiv:0712.0483.

[11] J. Biamonte and P. Love, "Realizable Hamiltonians for universal adiabatic quantum computers," Phys. Rev. A., vol. 78, p. 012352, 2008, arXiv:0704.1287.

[12] S. Bravyi, D. DiVincenzo, R. Oliveira, and B. Terhal, "The complexity of stoquastic local hamiltonian problems," Quantum Inf. Comput., vol. 8, no. 5, pp. 0361-0385, 2008, quant-ph/0606140.

[13] E. Farhi, J. Goldstone, S. Gutmann, and M. Sipser, "Quantum computation by adiabatic evolution," MIT, Tech. Rep. MIT-CTP-2936, 2000, quant-ph/0001106.

[14] M. Johnson, M. Amin, S. Gildert, T. Lanting, F. Hamze, N. Dickson, R. Harris, A. Berkley, J. Johansson, P. Bunyk, E. Chapple, C. Enderud, J. Hilton, K. Karimi, E. Ladizinsky, N. Ladizinsky, T. Oh, I. Perminov, C. Rich, M. Thom, E. Tolkacheva, C. Truncik, S. Uchaikin, J. Wang, B. Wilson, and G. Rose, "Quantum annealing with manufactured spins," Nature, vol. 473, no. 7346, pp. 194-198, 2011.

[15] S. Bravyi, A. Bessen, and B. Terhal, "Merlin-Arthur games and stoquastic complexity," 2006, quant-ph/0611021.

[16] S. Jordan, D. Gosset, and P. Love, "Quantum-Merlin-Arthur-complete problems for stoquastic Hamiltonians and Markov matrices," Phys. Rev. A., vol. 81, no. 3, p. 032331, 2010, arXiv:0905. 4755.

[17] S. Bravyi and M. Vyalyi, "Commutative version of the k-local Hamiltonian problem and common eigenspace problem," Quantum Inf. Comput., vol. 5, no. 3, pp. 187-215, 2005, quant-ph/0308021.

[18] M. Hastings, "Topological order at nonzero temperature," Phys. Rev. Lett., vol. 107, no. 21, p. 210501, 2011.

[19] T. Cubitt and A. Montanaro, "Complexity classification of local Hamiltonian problems," 2014, arXiv:1311.3161.

[20] A. Bookatz, "QMA-complete problems," Quantum Inf. Comput., vol. 14, no. 5\&6, pp. 361-383, 2014, arXiv: 1212.6312.

[21] A. Childs, D. Gosset, and Z. Webb, "The Bose-Hubbard model is QMAcomplete," 2013, arXiv:1311.3297.

[22] S. Bravyi, "Monte Carlo simulation of stoquastic Hamiltonians," 2014, arXiv: 1402.2295.

[23] J. Kempe, D. Bacon, D. Lidar, and K. B. Whaley, "Theory of decoherence-free fault-tolerant universal quantum computation," Phys. Rev. A., vol. 63, p. 042307, 2000, quant-ph/0004064.

[24] E. Lieb and D. Mattis, "Ordering energy levels of interacting spin systems," J. Math. Phys., vol. 3, p. 749, 1962.

[25] J. Vidal, S. Dusuel, and T. Barthel, "Entanglement entropy in collective models," Journal of Statistical Mechanics: Theory and Experiment, vol. 2007, p. P01015, 2007, cond-mat/0610833.

[26] R. Horodecki and M. Horodecki, "Information-theoretic aspects of inseparability of mixed states," Phys. Rev. A., vol. 54, no. 3, pp. 18381843, 1996, quant-ph/9607007.

[27] A. Bulatov, "A dichotomy theorem for constraints on a three-element set," in Proc. 43 ${ }^{\text {rd }}$ Annual Symp. Foundations of Computer Science, 2002, pp. 649-658.

[28] D. Aharonov and L. Eldar, "On the complexity of commuting local Hamiltonians, and tight conditions for topological order in such systems," in Proc. 52 $2^{\text {nd }}$ Annual Symp. Foundations of Computer Science, 2011, pp. 334-343, arXiv:1102.0770.

[29] J. Yan and D. Bacon, "The $k$-local Pauli Commuting Hamiltonians problem is in P," 2012, arXiv:1203.3906.

[30] N. Schuch, "Complexity of commuting Hamiltonians on a square lattice of qubits," Quantum Inf. Comput., vol. 11, no. 11\&12, pp. 901-912, 2011, arXiv:1105.2843.

[31] A. Childs, D. Leung, L. Mancinska, and M. Ozols, "Characterization of universal two-qubit Hamiltonians," Quantum Inf. Comput., vol. 11, pp. 19-39, 2011, arXiv:1004.1645.

[32] M. Bremner, R. Jozsa, and D. Shepherd, "Classical simulation of commuting quantum computations implies collapse of the polynomial hierarchy," Proc. Roy. Soc. Ser. A, vol. 467, no. 2126, pp. 459-472, 2011, arXiv:1005.1407. 